\title{
Tissot et la conception de la médecine savante au $18^{\mathrm{e}}$ siècle*
}

Par Lazare Benaroyo

\section{Résumé}

Le concept de médecine éclairée a été créé par une élite médicale savante du $18^{e}$ siècle, dont Tissot faisait partie. Cette médecine cherchait à se distinguer de sa contrepartie, la médecine populaire (cet adjectif émanait de cette même élite), par le fait qu'elle fondait son savoir sur un rationalisme critique qui s'appuyait sur l'observation et l'expérience, ainsi que sur leur évaluation numérique. Il est montré dans cet article comment Tissot, qui avait subi l'influence de Fr. Boissier de Sauvages, d'Albrecht von Haller et de J.G. Zimmermann, concevait l'observation et l'expérience en médecine. Il ressort de cette étude que la médecine savante, telle que notre auteur l'a dépeinte, semble bien se situer à l'origine de notre attitude scientifique moderne en médecine.

La tradition philosophique rationaliste constituait le principal fondement de l'univers intellectuel des hommes de science de la seconde moitié du $18^{\mathrm{e}}$ siècle européen. Suivant la voie ouverte deux siècles plus tôt par Galilée et Francis Bacon, et prenant la pensée newtonienne pour modèle, l'activité rationnelle cherchait alors à s'appuyer sur l'observation et l'expérience. Ce type de rationalité, que nous pourrions qualifier de critique, s'exprimait non seulement dans des œuvres canoniques qui scandaient sa gloire, mais aussi dans des écrits plus singuliers qui en éclairaient les marges: tels par exemple des traités médicaux, rédigés en français par des médecins qui, sans vouloir faire preuve d'érudition, cherchaient à dénoncer les erreurs dites populaires en matière de santé.

Ces ouvrages, qui contenaient des chapitres entiers décrivant la juste manière de comprendre et de combattre les maladies, devaient permettre au lecteur averti d'éviter de commettre des erreurs de traitement à l'aide d'explications simples et accessibles. Ils comprenaient également de longs

\footnotetext{
* Séminaire donné le 6 juin 1989 à l'Institut d'Histoire de la Médecine et de la Santé Publique à Lausanne.
} 
chapitres sur les charlatans et le charlatanisme, au sein desquels leurs auteurs, qui se fondaient sur un rationalisme critique, cherchaient ouvertement à légitimer leur propre savoir. En opposant leur science aux pratiques qu'ils qualifiaient eux-mêmes de populaires, et dont le charlatan était la principale figure emblématique, ces médecins se disant éclairés véhiculaient l'image d'un savoir-faire populaire qui apparaissait en tous points antinomique à la représentation d'une médecine savante qu'ils cherchaient à promouvoir. C'est ainsi qu'au travers de ces écrits singuliers, certains médecins des Lumières parvenaient à délimiter le champ et les marges de leur propre savoir, qu'ils pouvaient du même coup légitimer aux yeux du plus grand nombre. Nous trouvons des exemples de ce militantisme intellectuel dans les deux ouvrages suivants: le livre du Dr d'Iharce intitulé Erreurs populaires sur la Médecine, publié à Paris en 1783, et la très célèbre œuvre du Dr Samuel-Auguste Tissot, l'Avis au Peuple sur sa santé, dont l'édition originale a paru à Lausanne en $1761^{1}$. L'esprit des Lumières cherchait à s'incarner dans ces traités par le biais de la représentation d'un médecin qualifié d'éclairé, entendez éclairé par les lumières de la raison, appartenant de ce fait à un univers supérieur, entendez savant, souhaitant ainsi se distinguer d'une quelconque appartenance à un monde populaire. Le bon grain pourrait alors être séparé d'avec l'ivraie. Précisons cependant que les adjectifs «savant» et «populaire» tendaient à qualifier en ce siècle bien plus une attitude intellectuelle qu'une division sociale. Le Dr d'Iharce le précise d'ailleurs fort clairement dès les premières lignes de son ouvrage: «...quiconque livre sa raison aux erreurs populaires, écrit-il, quel que soit le rang qu'il tient dans le monde, est peuple à cet égard...». ${ }^{2}$ Le concept de médecine savante, et son corollaire, le concept de médecine populaire, qui tous deux voyaient le jour dans ces écrits singuliers, avaient des implications à la fois épistémologiques, sociologiques et politiques. Nous nous limiterons ici à l'étude des éléments qui les caractérisaient au plan de la théorie de la connaissance et de la méthodologie.

Tissot, nous l'avons vu, a activement participé à l'élaboration de ces notions. Ce natif de Grancy (village proche de Lausanne), a été à Montpellier l'élève de François Boissier de Sauvages (1706-1767), lui-même disciple de Thomas Sydenham (1624-1689). Boissier de Sauvages était à la fois clinicien, nosologiste et expérimentateur. Tissot a également entretenu une longue et solide amitié avec l'un des plus grands anatomistes, physiologistes et expérimentateurs de son temps, Albrecht von Haller (1708-1777) ${ }^{3}$. Ce dernier avait été l'élève à Leyden du célèbre Hermann Boerhaave 
(1668-1738), avant de devenir lui-même professeur à Göttingen après des études entreprises à Oxford, Paris, Strasbourg et Bâle. Gràce à ses relations avec Boissier de Sauvages et Albrecht von Haller, Tissot a été sensibilisé aux pensées anglaise et germanique; il a également acquis un savoir étendu, probablement représentatif du niveau supérieur des connaissances médicales de son époque. Il a défendu tout au long de sa carrière, au nom de ce savoir, une conception de la médecine éclairée que nous allons maintenant étudier plus en détail.

«Les plus grands médecins», écrit-il dans l'Avis au peuple sur sa santé, «ces hommes rares, qui, nés avec les plus heureux talents, ont éclairé leur esprit dès leur plus tendre enfance; qui ont cultivé ensuite avec soin toutes les parties de la physique; qui ont sacrifié les plus beaux moments de leur vie à une étude suivie et assidue du corps humain, de ses fonctions, des causes qui peuvent les empêcher, et de tous les remèdes; qui ont surmonté le désagrément de vivre dans les hôpitaux, parmi des milliers de malades; qui ont réuni à leurs propres observations, celles de tous les temps et de tous les lieux; ces hommes rares [...] ne se trouvent pas même tels qu'ils voudraient être, pour se charger du précieux dépôt de la santé humaine». ${ }^{4}$

Le médecin éclairé fonde ainsi, pour Tissot, une grande partie de son savoir sur le modèle de la physique, entendez newtonienne, c'est-à-dire sur une activité rationnelle s'appuyant sur l'observation et l'expérience. Que signifient ces termes?

Pour les médecins des Lumières, et en particulier pour ceux qui comme Tissot ont lu le Traité de l'expérience en général et en particulier dans l'art de guérir de J.G. Zimmermann, paru dans sa première édition en langue allemande en 1763, l'observation consiste à constater et à collecter des faits naturels en se fondant sur les témoignages des sens. L'observation médicale se pratique au chevet du patient, dans la salle d'autopsie ou dans le cadre d'une expérimentation effectuée sur des animaux ou des hommes. Pour ces mêmes lecteurs, l'expérience apparaît comme un nouvel instrument de connaissance, plus élaboré que l'observation. Elle est le lieu où le produit de la raison peut être mis à l'épreuve des faits; c'est grâce à elle que peuvent se déterminer des relations causales universellement valables entre des phénomènes; elle est devenue, depuis Newton, le support nécessaire à la découverte des lois naturelles, lois qui permettent à l'homme de déchiffrer le langage du grand livre de la Nature.

Le $18^{\mathrm{e}}$ siècle réalise l'importance de l'expérience en médecine: c'est en effet en se fondant sur ces lois que le médecin parviendra à déterminer, par voie inductive, avec confiance et sécurité, la cause naturelle, et souvent 
cachée, du phénomène morbide qu'il observe ${ }^{5}$. L'expérience raisonnée, unie à l'observation, est ainsi reconnue comme étant une des conditions nécessaires de la connaissance. Pour porter ses fruits, l'expérience doit cependant être conduite selon certaines règles. Tissot y adhère, comme en témoigne la plus grande partie de son œuvre. Examinons plus en détail quelles sont ces règles.

1) Dans une expérience, la cause doit être phénoménale, proportionnelle à l'effet observé ${ }^{6}$. La causalité finale doit être rejetée, et la causalité efficiente retenue. Tissot insiste sur ce point dans son Discours préliminaire à la Dissertation sur les parties irritables et sensibles des animaux (1755), l'œuvre maîtresse d'Albrecht von Haller qu'il a traduite en français à la demande de l'auteur.

2) Le nombre de causes doit être réduit autant que possible aux causes essentielles, en regard de la diversité possible des effets. Dans la Lettre à $\mathrm{Mr}$ Zimmermann contenant des observations sur la maladie noire... (1760), Tissot montre par le biais d'une autopsie que les signes distinctifs (les effets) de la maladie noire tels les vomissements et les évacuations rectales de sang, sont à mettre en relation causale avec une seule affection prenant son origine dans l'estomac; une phlegmasie est ici la cause commune des symptômes, contrairement à l'opinion des Anciens.

3) Une relation de cause à effet doit être observée de manière constante et uniforme dans le plus grand nombre de cas possibles pour pouvoir être considérée comme universellement valable, et ainsi élevée au rang de loi naturelle. Quelle cela signifie-t-il?

Depuis l'Antiquité gréco-latine et arabe, les médecins ont invoqué de longues séries d'observations pour démontrer leur propositions. A la fin du $3^{\mathrm{e}}$ siècle avant J.-C., la secte des Empiriques semble bien avoir reconnu que la sécurité de ses affirmations augmentait avec le nombre de ses observations ${ }^{7}$. Au $17^{\mathrm{e}}$ siècle, Giorgio Baglivi (1668-1706), puis au $18^{\mathrm{e}}$ siècle, Giovanni Battista Morgagni (1682-1771) et Albrecht von Haller ont avancé de très nombreuses expériences à l'appui de leurs conclusions, Morgagni dans ses recherches anatomiques et Haller dans ses études physiologiques. Ce dernier rapporte plus de 557 expérimentations dont 190 pratiquées par lui-même sur des animaux dans sa célèbre Dissertation sur les parties irritables et sensibles des animaux $(1755)^{8}$. David Hume (1711-1776) a établi au milieu du $18^{\mathrm{e}}$ siècle les fondements philosophiques de cette méthodologie ${ }^{9}$. Ce ne sera que bien plus tard, vers le milieu du $19^{\mathrm{e}}$ siècle, que cette approche se basant sur la multiplication nécessaire du nombre d'observations, trouvera un appui 
auprès de la théorie des probabilités qui lui permettra de déterminer avec précision les règles de son emploi. De cette union naîtra la statistique médicale que Jules Gavarret développera dans son célèbre ouvrage paru en $1840^{10}$. Si le mot statistique n'est pas encore couramment employé au $18^{\mathrm{e}}$ siècle, la chose est cependant déjà connue et pratiquée, comme nous allons maintenant le montrer.

La théorie des probabilités, théorie purement mathématique à ses débuts, dont on attribue la paternité à deux penseurs du $17^{\mathrm{e}}$ siècle, Pascal et Fermat, cherche au $18^{\mathrm{e}}$ siècle à déborder des mathématiques pour s'appliquer tout d'abord aux sciences morales, puis, vers la fin du siècle, aux sciences de la nature. Il s'agit là de projets formulés pour les sciences morales par Jakob Bernoulli (1654-1705) ${ }^{11}$ et pour les sciences morales et les sciences de la nature par Pierre-Simon Laplace (1749-1827). Durant tout le $18^{\mathrm{e}}$ siècle, les débats portant sur l'inoculation de la petite vérole illustrent également ce fait, puisqu'on y découvre des médecins, des mathématiciens et des philosophes qui fondent leurs principaux arguments sur des données tirées du calcul des probabilités; ainsi par exemple la querelle entre Daniel Bernoulli (1700-1782), favorable à l'inoculation, et Jean Le Rond d'Alembert (1717-1783) qui ne se laisse pas convaincre par les calculs de ce dernier ${ }^{12}$.

Comment parvenir à une certitude concernant un phénomène en s'appuyant sur la théorie des probabilités? Comment avoir confiance dans les conclusions d'une expérience? Tel est alors l'enjeu du débat. Les médecins favorables à l'inoculation cherchent à multiplier le nombre d'observations afin d'accroître le degré de certitude de leurs conclusions. Cette attitude dérive nécessairement, sans que cela ne soit pourtant clairement exprimé, de la théorie des probabilités, dans la mesure où cette théorie postule une relation directe entre le degré de certitude et le nombre d'observations: plus ce nombre est grand, plus le degré de certitude concernant la réalité d'un phénomène observé est élevé. Un degré de certitude très élevé équivaut alors à une preuve. Une certitude ayant valeur de preuve est qualifiée de «certitude morale» ${ }^{13}$. Une telle représentation de la certitude postule, conformément à l'esprit du siècle, une finitude de la pensée humaine, pensée dont la richesse consiste en sa capacité de déterminer sa propre marge d'erreur. Leibniz, Jakob Bernoulli et David Hume ont apporté des contributions épistémologiques marquantes à ce propos. La certitude morale, sur laquelle peuvent dès lors s'appuyer les savants lors de leurs recherches sur les phénomènes naturels, est bien entendu moins précise que la certitude démonstrative, absolue, qui découle de l'usage des mathématiques pures. 
Elle est cependant considérée par David Hume et P.J.G. Cabanis (1757-1808) comme «suffisante pour assurer la conservation et le bien-être de l'espèce humaine» ${ }^{14}$. C'est en se fondant sur cette représentation de la certitude, propre au siècle des Lumières - et que nous admettons encore de nos jours - que la science statistique se développera au $19^{\mathrm{e}}$ siècle.

Tissot vit donc a une époque où les concepts de probabilités et de certitude morale semblent être d'usage courant dans les cercles érudits. Le catalogue manuscrit de sa bibliothèque ${ }^{15}$ nous permet d'ailleurs de constater qu'il possède certains ouvrages auxquels il vient d'être fait référence. Il écrit d'autre part dans L'Inoculation justifiée (1754): «Toute notre vie ne roule que sur des probabilités [...] dans toute les matières susceptibles de calcul, et où l'on peut nombrer les raisons, c'est le calcul qui doit nous déterminer» ${ }^{16}$. Il défend le concept de certitude morale dans son Discours préliminaire à l'ouvrage de Haller: «Le Pirrhonisme qui nie toute certitude, et celui qui n'admet que la certitude géométrique, sont également ridicules et dangereux, écrit-il; les inductions ont leurs règles, et les propositions qu'on découvre en les suivant exactement, ont le même degré de force, que les propositions mathématiques les plus rigoureusement démontrées» ${ }^{17}$.

Tissot fait de plus référence à la théorie des probabilités à plusieurs reprises dans son œuvre: il s'appuie sur des dénombrements ainsi que sur un calcul des probabilités sommaire dans l'argumentation qu'il développe dans L'Inoculation justifiée en faveur de l'introduction de l'inoculation de la petite vérole ${ }^{18}$. Les dénombrements qu'il relate lui permettent de démontrer les points suivants: 1) la petite vérole inoculée préserve de la petite vérole naturelle, 2) l'inoculation ne provoque pas de décès, 3) l'inoculation permet de réduire le taux de mortalité de la petite vérole naturelle ${ }^{19}$. Ainsi la chance de survie est plus grande après l'inoculation; il est dès lors démontré que cette méthode est efficace. Quelles raisons permettent d'expliquer cette efficacité? Par l'intermédiaire de cette méthode, le médecin provoque une petite vérole artificielle bien moins grave que la petite vérole naturelle. Cela est attesté par le fait que la mortalité observée après inoculation est plus faible et que le nombre de «boutons» (pustules) est beaucoup moins élevé. Le médecin pouvant choisir le moment d'inoculer le malade (préparation), il évite les facteurs qui donnent habituellement à la petite vérole son caractère grave, voire mortel: tels un tempérament affaibli, une maladie intercurrente, des passions excessives, la saison, la constitution régnante, l'exercice violent ou encore la débauche ${ }^{20}$. Voilà ainsi démontrée l'efficacité d'une méthode préventive, l'inoculation de la petite vérole; les facteurs qui déterminent 
cette efficacité semblent contrôlables, et le pouvoir prédictif du médecin semble accru. Nous trouvons là les principaux critères de validation qui seront plus tard considérés comme étant susceptibles de conférer un caractère scientifique aux recherches cliniques.

D'autres écrits attestent encore que Tissot estime indispensable de multiplier le nombre d'observations. Ainsi écrit-il dans la Dissertation sur les fièvres bilieuses (1758):

«La médecine, dans sa première enfance, n'était dirigée que par un petit nombre d'observations, et les observations isolées, souvent imparfaites, nous induisent en erreur; mais lorsqu'ensuite ces observations sont devenues extrêmement nombreuses, elles ont été rassemblées par des hommes pleins de sagacité et d'érudition, et ces hommes ont su déduire des résultats et des règles qui sont les véritables éléments de la science médicale, et la pierre de touche, au moyen de laquelle on pourra, dans tous les âges, distinguer l'exacte vérité, de ce qui n'en a que l'apparence». ${ }^{21}$

Après avoir étudié les caractéristiques de la médecine savante d'un point de vue théorique, portons notre attention sur le savoir-faire du médecin éclairé. Le lecteur familier des textes du $18^{\mathrm{e}}$ siècle ne sera pas étonné de constater que chez Tissot, comme chez la plupart des auteurs d'ouvrages médicaux de cette époque, les réalisations ne sont pas toujours à la hauteur des ambitions exprimées. C'est ainsi par exemple, que dans l'Avis au peuple sur sa santé, notre auteur recommande des traitements qui s'identifient encore bien souvent aux méthodes curatives populaires qu'il dénonce justement dans ce même ouvrage ${ }^{22}$. Il faut bien avouer cependant qu'il n'était pas le seul homme de science de son temps à éprouver de telles difficultés. Comment parvenir à une réelle efficacité dans un univers où, comme l'a récemment écrit Jacques Roger, les faits n'étaient pas aisés à isoler, où les expériences n'étaient pas facile à conduire, où la méprise entre un fait et une conjecture était fréquente ${ }^{23}$ ? La méfiance était en effet de règle, faute de pouvoir systématiquement vérifier; «la vérification des faits par une répétition des expériences n'était pas toujours aisée, précise Jacques Roger. La science était en train de découvrir l'expérimentation et de s'apercevoir qu'il ne suffisait pas de regarder pour voir» ${ }^{24}$. Les méthodes de vérification n'étaient en fait pas encore disponibles ${ }^{25}$. L'ensemble de l'œuvre de Tissot confirme bien la règle. C'est peut-être dans le domaine préventif, la défense de l'inoculation de la petite vérole, que se trouve l'exception, et qu'une ébauche de réalisation de ses ambitions est perceptible.

Malgré les difficultés pratiques qu'elle éprouve à se réaliser, la médecine éclairée, telle que Tissot la conçoit, semble bien se situer, dans son principe 
théorique et dans son projet, à l'origine de notre attitude scientifique moderne en médecine. Cette attitude rend la connaissance de la nature en grande partie dépendante de la méthode statistique mathématique s'appuyant elle-même sur la théorie et le calcul des probabilités. Or, comme nous l'avons démontré, cette méthode avait déjà cours sous forme embryonnaire au $18^{\mathrm{e}}$ siècle. Tissot y fait largement référence dans son Discours préliminaire à la célèbre œuvre de Haller parue en 1755, ainsi que dans divers écrits de jeunesse tels par exemple L'inoculation justifiée (1754) ou la Dissertation sur les fièvres bilieuses (1758). Il recourt abondamment à cette méthode dans sa défense de l'inoculation de la petite vérole.

La méthode statistique a connu depuis cette époque un essort considérable, au point d'être devenue aujourd'hui indispensable à la recherche en biologie et en médecine. Notre engouement actuel pour la médecine scientifique a peut-être tendance à nous en faire oublier les origines. Comment résister alors au désir de faire sortir de l'oubli ce merveilleux texte de PierreSimon Laplace, datant de 1815:

«Le calcul des probabilités, nous dit-il, peut faire apprécier les avantages et les inconvénients des méthodes employées dans les sciences conjecturales. Ainsi, pour reconnaître le meilleur des traitements en usage dans la guérison d'une maladie, il suffit d'éprouver chacun d'eux sur un même nombre de malades, en rendant toutes les circonstances parfaitement semblables: la supériorité du traitement le plus avantageux se manifestera de plus en plus, à mesure que ce nombre s'accroîtra; et le calcul fera connaître la probabilité correspondante de son avantage, et du rapport suivant lequel il est supérieur aux autres». ${ }^{26}$

N'est-ce pas là une réelle préfiguration des essais cliniques contrôlés sur lesquels notre thérapeutique s'appuie depuis les années 1930? 


\section{Notes}

1 Voir Benaroyo L.: L'Avis au peuple sur sa santé de Samuel-Auguste Tissot (1728-1797): la voie vers une médecine éclairée. Zürcher medizingeschichtliche Abhandlungen Nr. 195, Juris Verlag, Zürich, 1988.

2 D'Tharce: Erreurs populaires sur la Médecine, Paris 1783, p. i.

3 Voir Milt B.: «Empirie und das statistisch fundierte biologisch-medizinische Denken in der Geschichte», Gesnerus 13: 1-28 (1956), p. 13.

4 Tissot S.A.: L'Avis au peuple sur sa santé, Lausanne 1766, p. 611.

5 Pour plus de détails sur les notions de cause et d'induction, voir les deux articles suivants: King L.: «Some problems of causality in eighteenth-century medicine», Bull. Hist. Med. 37: 15-24 (1963);

King L.: «Evidence and its evaluation in eighteenth-century medicine», Bull. Hist. Med. 50: 174-190 (1976).

6 Voir Hume D.: Enquête sur l'entendement humain. Traduit de l'anglais par Didier Deleule,

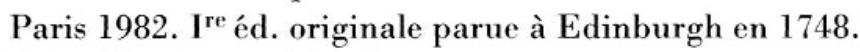

7 Voir Milt B.: op. cité p. 3.

8 Voir Milt B.: op. cité p. 13. Voir également Haller A.: Dissertation sur les parties irritables et sensibles des animaux, Lausanne 1755, p. 1.

9 Voir Hume D.: op. cité.

10 Gavarret J.: Principes généraux de statistique médicale, Paris 1840.

11 Voir à ce propos l'excellent ouvrage suivant: Hacking I.: The Emergence of Probability, Cambridge University Press, 1975 pp. 143 ss.

12 Voir Bernoulli D.: Essai d'une nouvelle analyse de la mortalité causée par la petite vérole et des avantages de l'Inoculation pour la prévenir. In: Mémoires de mathématiques et de physique de l'Académie Royale des Sciences, Paris 1760, p. 1-45.

Voir également Bernoulli D.: «Réflexions sur les avantages de l'Inoculation», Mercure de France, juin 1760 .

Voir également d'Alembert J. le Rond.: Opuscules mathématiques, tomes 2 et 4, Paris, 1762 et 1768.

13 Voir Hacking I.: op. cité p. 145-146.

14. Cabanis P.J.G.: Du degré de certitude de la médecine, Paris 1798, p. 126.

15 Ce manuscrit se trouve à la bibliothèque cantonale universitaire de Lausanne sous l'inscription suivante: MSS, IS 3784 no 71.

16 Tissot S.A.: L'Inoculation justifiée, Lausanne, 1754, p. 87-88.

17 Tissot S. A.: Discours préliminaire à la Dissertation sur les parties irritables et sensibles des animaux (Haller 1755), p. XLI.

18 Tissot 1754, op. cité p. 10-11.

19 Tissot ibid. p. 26.

20 Tissot ibid. p. 34 ss.

21 Tissot S. A.: Dissertation sur les fièvres bilieuses, et histoire de l'Epidémie Bilieuse qui régna à Lausanne en 1755, Paris 1799-1800. Traduit du latin avec quelques additions par Maurice Mahot. L'édition originale en latin a paru à Lausanne en 1758. La citation est tirée de l'édition française, p. 22.

22 Voir Benaroyo L.: op. cité p. 50-51. 
23 Roger J.: Les sciences de la vie dans la pensée française du $18^{e}$ siècle, A. Colin, $2^{\mathrm{e}}$ édition, Paris, 1971, p. 184 ss.

24. Roger J.: ibid. p. 193.

25 Voir King 1976, p. 188.

26 Laplace P.S.: Essai philosophique sur les probabilités, $5^{\mathrm{e}}$ éd. de 1825, Ed. Chr. Bourgeois, Paris 1986, p. 116-117.

\section{Summary}

\section{Tissot and the concept of learned medicine in the 18th century}

The notion of enlightened medicine was conceived by an elite group of eighteenth century medical scholars to which Tissot belonged. This elite group sought to create an identity separate from its counterpart, that is to say popular medicine as defined by this group. The distinctive characteristic of this enlightened medicine was its critical rationalism based on observation and experiment as well as their numerical evaluation. This article describes how Tissot, influenced by Fr. Boissier de Sauvages, Albrecht von Haller and J.G.Zimmermann, understood observation and experiment in medicine. This study shows that learned medicine, as portrayed by our author, seems to have originated today's modern scientific medical approach.

\section{Zusammenfassung}

\section{Tissot und der Begriff der wissenschaftlichen Medizin im 18. Jahrhundert}

Der Begriff der aufgeklärten Medizin wurde im 18. Jahrhundert von einer Elite medizinischer Gelehrter geschaffen, der auch Tissot angehörte. Diese Medizin wollte sich von ihrem Gegenstück, das von derselben Elite als Volksmedizin bezeichnet wurde, absetzen, indem sie ihr Wissen auf einen kritischen Rationalismus abstützte, der auf Beobachtung und Experiment sowie deren zahlenmäßiger Bewertung beruhte. In diesem Artikel wird gezeigt, wie Tissot, der von Fr. Boissier de Sauvages, Albrecht von Haller und J.G.Zimmermann beeinflußt war, Beobachtung und Experiment in der Medizin auffaßte. Aus dieser Studie geht hervor, daß die gelehrte Medizin, wie sie Tissot beschrieben hat, am Ursprung unserer modernen, wissenschaftlichen Haltung in der Medizin stehen dürfte.

Dr Lazare Benaroyo

Avenue de la Gare 7

CH-1003 Lausanne 